\title{
Emission-free driving
}

Gunnar-Marcel Klein

MANN+HUMMEL International GmbH \& Co. KG

This manuscript is not available according to publishing restriction. Thank you for your understanding. 